\title{
Assessment of body composition in oncologic patients: Experimental survey on the role of bioimpedentiometric analysis
}

\author{
Maria Alessandra Gammone ${ }^{1,2}$, Cristiana Ficoneri ${ }^{1}$, Nicolantonio D'Orazio ${ }^{1}$ \\ 1. Department of Oral Medical and Biotechnological Science, University of Chieti-Pescara, Italy \\ 2. E-mail any correspondence to: m.alessandra.gammone@gmail.com
}

\begin{abstract}
Overall survival of oncologic patients is strongly influenced by the incidence of malnutrition, with subsequent loss of muscle mass until sarcopenia. In this respect, the assessment of body composition has a pivotal role in order to manage the clinical consequences of muscle loss.

Aim: This study focuses on the body composition assessment in oncologic patients, following a diet plan in order to detect and contrast neoplastic cachexia.

Materials and Method: 35 oncologic patients were enrolled and divided into two groups: 24 responders (R) and 11 non-responders (NR). Anthropometric data were collected and body composition was assessed through bioimpedentiometry. All patients received an individualized normocaloric diet. Energetic content was assigned on the basis of individual basal metabolic rate estimated by BIA. All patients were revaluated by anthropometry and bioimpedentiometry 3 and 6 months thereafter.

Results: Comparing the two male groups $\mathrm{R}$ and NR after 6 months, the former maintained almost the same confidence interval, unlike the latter whose interval increased, indicating a light worsening both of body composition and of clinical conditions. Furthermore, in the same male responder group, an improvement of the phase angle ( $\mathrm{PhA})$, a positive prognostic factor, was found, with an average weight loss between 2,6 - $3 \mathrm{~kg}$ of fat mass (FM). Besides, data show how the $\mathrm{R}$ female group maintained the confidence interval unlike the NR female group, whose confidence interval remarkably increased, therefore indicating an important alteration of body composition and subsequently a clinical conditions worsening. Finally, comparing the NR male group with the NR female group after 6 months, a remarkable worsening of body composition (marker of tissue decay and damage) could be noticed in the latter group.
\end{abstract}

Conclusion: After 6 months, NR patients show worse results (both in the body composition and in the clinical conditions) compared to the responders, with particularly worse results in females, probably because of a genetically determined sex-related smaller muscle cells and inferior muscular strength. A multidimensional assessment of oncologic patient is necessary, with a special attention to nutritional evaluation and body composition monitoring, in order to avoid malnutrition and subsequent further clinical worsening.

Keywords: Bioimpedance; nutrition; malnutrition; oncologic diseases

\section{Introduction}

Malnutrition is a multifactorial syndrome associated to advanced tumors and it negatively affects the quality of life, representing the cause of death for about $20 \%$ of the affected patients in the last stage [1]. Evidence shows that malnutrition, anorexia, appetite and weight loss are common in patients with cancer since the initial stages of the illness. This is generally due to the onset of symptoms such as early satiety, taste changes, nausea, vomiting, food aversion, altered taste and smell disorders [2]. Therefore, malnutrition should no longer be considered an inevitable side effect of the disease, rather than a foreseeable and reversible occurrence. Consequences of weight loss have a remarkable effect on the patient's health causing an increased toxicity on healthy cells and making tumoral cells more resistant to the treatment [3]. At the same time, the immune defense's weakening increases the frequency of 
hospital stays and worsens prognosis, elevating the death rate. Malnutrition in oncologic patients is remarkable [4] because of the multiple and serious consequences associated with the illness, including anorexia, cachexia and sarcopenia. This determines a reduced physical function [5], very often influenced by the presence of mucositis, hemesis, diarrhea and malabsorption, globally bringing to a reduced nutrients intake [6]. When the inflammatory cachectic state causes muscle mass depletion and physical functionality loss, sarcopenia is configured. For all these reasons neoplastic cachexia can be defined as a multifactorial syndrome characterized by a progressive muscle mass loss (with or without fat mass loss), leading to a functional damage which can harmfully affect prognosis and compromise quality of life [3]. From a clinical point of view, we have cachexia in presence of an involuntary weight loss of more than $5 \%$ in the previous 6 months or with respect to pre-illness, or of more than $2 \%$ if the patient's $\mathrm{BMI}$ is $<20 \mathrm{~kg} / \mathrm{m}^{2}$ or if sarcopenia is concomitant [3]. For cachexia diagnosis, in addition to weight loss three of the following pathological conditions are needed: anorexia, fatigue, or reduced muscle strength and total fat free mass, biochemical alterations (increase in inflammation indexes, such as C-reactive protein or inflammatory cytokines, anemia, hypoalbuminemia) [7].

From a pathophysiological perspective instead, cachexia occurs with a negative metabolic balance both energetic and proteic, caused by a reduced caloric intake combined with immunologic disorders [8]. Illness progression contributes to increased energetic expenditure; this in turn contributes to activation of the immune system. The impairment of the system contributes to the loss of body mass, particularly muscle mass, resulting in a proteolysis enhancement, with a subsequent reduction in the protein synthesis, a lipolysis enhancement with adipose tissue stores loss and eventually also an enhanced gluconeogenesis, due to the mediators produced by the tumor itself. Cachexia is very often underestimated for various reasons, as for example when it is associated with a high prevalence of overweight. A recent study [9] has actually shown that fat mass excess, with high body mass index (BMI) value, reduces the reliability of the nutritional state assessment in oncologic patients.

Therapeutic intervention should aim to the improvement of the patient's clinical condition, with particular attention to metabolic and nutritional state, in order to improve the patient's general outcome $[10,11]$.

\section{Materials and methods}

Subjects: 35 consecutive oncologic patients ( 21 women, 14 men, average age 53 years, range $34-67$ ) were enrolled at the Human and Clinical Nutrition Unit of "G. d'Annunzio University" of Chieti. Patients were divided into two groups: Responders (R) $\mathrm{n}^{\circ} 24$ (16 women and 8 men) and nonresponder (NR) $n^{\circ} 11$ ( 5 women and 6 men). The compliance to the proposed diet was considered as inclusion criteria.
Study design: All patients received an individualized normocaloric diet plan. Total kilocalories were established based on basal metabolic rate estimated by bioimedentiometry. Macronutrient distribution was determined based on the patients' comorbidities.

All patients were evaluated at baseline (TO) and reevaluated by anthropometric analysis and bioimpedentiometry after 3 months (T3) and 6 months thereafter (T6). Anthropometric data and BMI was calculated using weight and height measured values.

Body composition was assessed and evaluated through bioimpedentiometric analysis (BIA). This technique uses the following theory: when an electric current is applied to a cylinder filled with a saline solution, the fluid opposes to the current, the higher its electrical resistance ( $R$ expressed in ohm). Considering the human body as a series of connected cylinders, the measured resistance reflects the body composition. Regarding the measurement, four electrodes are placed on the skin: a couple on the back of the hand and a couple over the instep. Hand and feet should belong to the same side of the patient's body. The subject lies supine with his legs slightly apart and upper limb away from the body. The device generates a low intensity alternate current, which flows into the patient's body. Final data are expressed in kilograms and in percent of total body weight.

Impedentiometric data were collected from the instrument BIA 101 (Akern Bioresearch srl, Italy), and processed by the related software BIVA SOFTWARE 2002.

BIA allows the assessment of body composition, defining total body water (TBW), intracellular water (ICW), extracellular water (ECW), fat free mass (FFM), body cell mass (BCM), fat mass (FM).

Using a non-invasive instrument, it is possible to determine the electrical impedance, a vector quantity representing the oppositional force to the flow of an alternating current through body tissues. BIA is based on the principle that human body impedance ( $\mathrm{Z}$ ) is inversely proportional to body water and electrolytes content, when body receives an alternating current $(A C)$ : current runs through extracellular fluids at low frequencies, while overcomes cellular membranes penetrating intracellular fluids at high frequencies. BIA offers more precision and reliability in measuring adiposity than anthropometric methods (plicometry and body circumferences) even if these provide useful information related to fat mass distribution. The use of bioimpedentiometry in oncology allows to accurately monitor the patient's nutritional state and has proved to be a valid instrument improving compliance to dietetic prescriptions. In biological systems, electrical conduction is linked to water distribution: as body fat free mass contains the greatest part of water and electrolytes, conductivity is greater than in fat mass.

Bioimpedentiometric vector analysis is very useful in order to investigate body hydration, represented by the impedance vector length (the vector lengthening is 
associated to a dehydration state, the shortening to fluid retention) and nutritional state, which is associated with phase angle values; the greater the phase angle the higher the cellular mass. Conversely, the minor inclination of the impedance vector is an indicator of a worse nutritional state.

BIA is considered a very useful technique to monitor hydration and nutritional state of different kinds of patients in serious clinical conditions, such as oncologic ones: it can be very helpful in cancer patient management, particularly to foresee some consequences of the disease, such as malnutrition and cachexia. For this reason, the European Society of Parenteral and Enteral Nutrition (ESPEN) considers it a rapid and non-invasive method to estimate body composition in the context of nutritional evaluation, recognizing its strong prognostic value. One of the detected parameters is the phase angle $(\mathrm{PhA})$, representing an indicator of global health state. In the last decade, several studies have investigated on the role of $\mathrm{PhA}$ as a prognostic tool and as an indicator of cell membrane function and nutritional state in various pathological conditions, such as cancer. The link between hydration and $\mathrm{PhA}$ is more evident in disease-linked malnutrition, especially in oncologic patients, where we can find an increase in intracellular water, with consequent changes in PhA [12].

$\mathrm{PhA}$ value depends on age, gender and BMI: at low values a decrease in cellular integrity has been observed, even leading to cellular death: on the contrary, a greater cell integrity has been observed at high values [13]. Survival strictly depends upon cell integrity and several studies on patients with different kind of tumors have shown that at a low PhA value a diminished probability of survival corresponds $[14,15]$. Patients who exibited a $\mathrm{PhA} \leq 5.6^{\circ}$ had a survival expectancy of about 23 months, compared to patients with a higher PhA, whose survival was 49.9 months on average. In both cases, early nutritional interventions have been proposed for those patients showing values below the limit point, however currently no nutritional therapy has actually warranted survival time prolongation.

Another association between PhA and malnutrition seems to emerge: a study showed that a PhA value $<5^{\circ}$ is indicative of an inadequate caloric intake, which can also be confirmed by low plasmatic albumin values. In addition to being a malnutrition indicator hypoalbuminemia in fact alters ECW/ICW ratio leading to an underestimation of the $\mathrm{PhA}$ value. In light of this, it appears evident that the bioelectrical impedance vector analysis BIA is a fundamental tool to assess first of all nutritional state but also hydration state: parameters strictly related to $\mathrm{PhA}$ value, are widely recognized as prognostic factors in altered health states. There are no complex mathematical equations involved in BIA technology: this device simply uses the resistance and the reactance at $50 \mathrm{kHz}$ and it is normalized to the subject's height. Once the measurement is performed, the subject's result are illustrated in the form of a "dot on the vector". The position of this dot reflects the subject's health status in comparison to the subject's chosen demographic group. Indeed, the treatment aim should be weight and muscle mass regain, along with the improvement of the patient's functional capacity and quality of life. Oncologic patients more frequently exhibit nutritional disorders also in the early stages of illness such as after surgery. Among cancer patients losing body weight, actually $20-30 \%$ die due to direct or indirect consequences of malnutrition [16]. Many factors can influence cancer-related malnutrition development and weight loss. They can be attributed to either the disease itself (malabsorption, location of the tumor or metastasis) or to the therapeutic intervention (chemotherapy, radiotherapy, surgery) which very often involves symptoms such as anorexia, nausea, vomit, dysphagia, and can determine an alteration or a worsening of the nutritional state.

Psychological issues can also negatively impact the food intake, worsening the patient's state. It is therefore evident that nutritional intervention should be a timely integrant part of the whole oncologic treatment. At the same time, it should be individual-tailored, targeting malnutrition prevention and treatment. Nutritional therapy can have a preventive value (if the patient has been followed since diagnosis) or a supporting role during treatment: the aim is to minimize or avoid nutritional state impairment during therapy in order to improve the deterioration of physical conditions. A follow-up with nutritional assessment should be performed every time significant weight loss alterations occur: particularly, body weight and BMI, body composition estimate and food intake analysis are all tools allowing the evaluation of eventual early signs of malnutrition in cancer patient.

Considering the epidemiological data on malnutrition and its complications, a greater awareness leading to cultural and therapeutic interventions on the issue is needed. The more recent scientific evidence shows how body composition assessment at the moment of cancer diagnosis, is the most valid therapy to prevent malnutrition. The aim of our study was to evaluate in oncologic patients, the efficacy of body composition evaluation and diet plan on weight maintenance and on body composition alterations measured by BIA and assessed with the BIVA method previously described. Obtained data have been compared to the ones obtained from patients who did not follow the diet.

\section{Ethical approval}

The research related to human use has been complied with all relevant national regulations, institutional policies and in accordance with the tenets of the Helsinki Declaration, and has been approved by the authors' institutional review board or equivalent committee.

Informed consent

Informed consent has been obtained from all individuals included in this study. 
Results

Results show that, at the confidence interval between $0-6$ months, $\mathrm{R}$ maintains the same confidence interval unlike the NR, whose confidence interval increases, so showing a slight worsening both in body composition and in clinical conditions (Fig.1).

In male $\mathrm{R}$, a PhA improvement (positive prognostic factor) with an average weight loss of $2.6-3 \mathrm{~kg}$ of fat mass is visible.

Female oncologic patients at T0 compared to T6 (Fig.2): Results show that, at the confidence interval between $0-6$ months $\mathrm{R}$ maintain the same confidence unlike the NR whose confidence interval remarkably increases, so showing a serious worsening in clinical conditions.

Compared to the previous male patients' graph, this data show how NR female move toward a sharper deterioration compared to NR male, during the 6 months observation. Another important aspect emerging from the results is that during nutritional monitoring, subjects lose weight without any change in soft tissues and maintain a steady hydration. This is a positive element, excluding the neoplastic cachexia, where weight loss, particularly regarding fat free mass, would determine an important change in total body water.
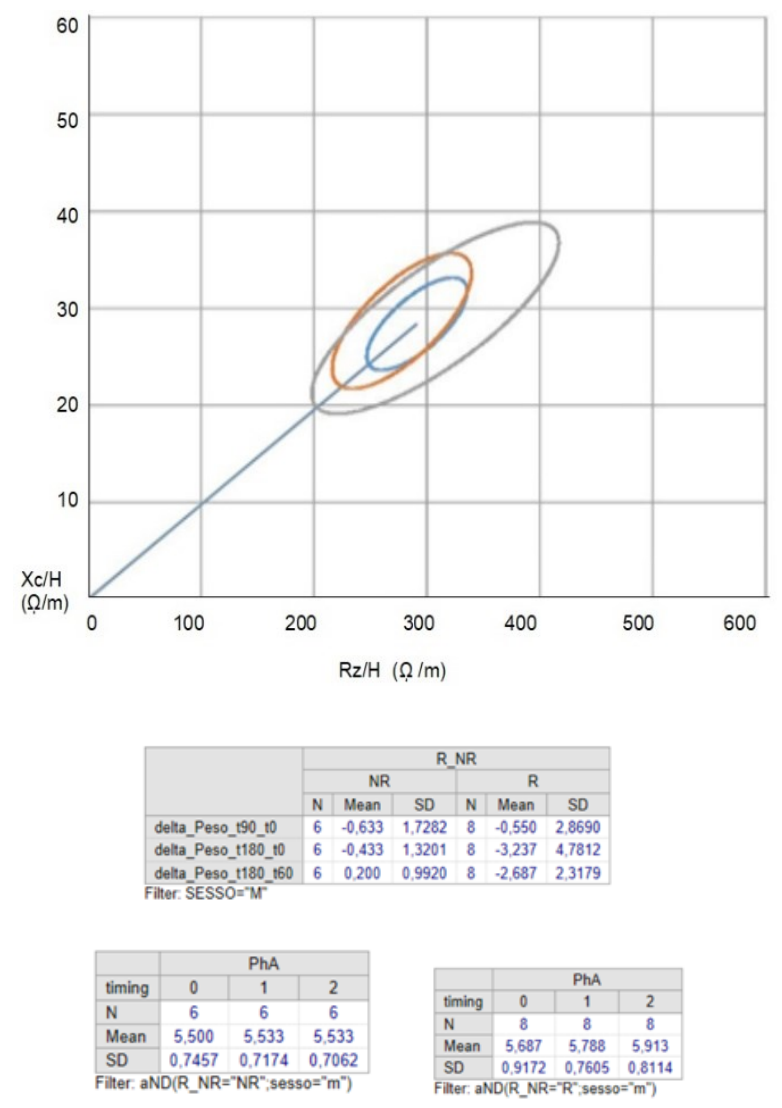

Fig.1: The graph compares male oncologic patients, both $\mathrm{R}$ and NR, at T0 (baseline) and T6 (evaluation after 6 months). Male oncologic patients at T0 compared to T6: $R$ are marked by orange color and NR by grey color.

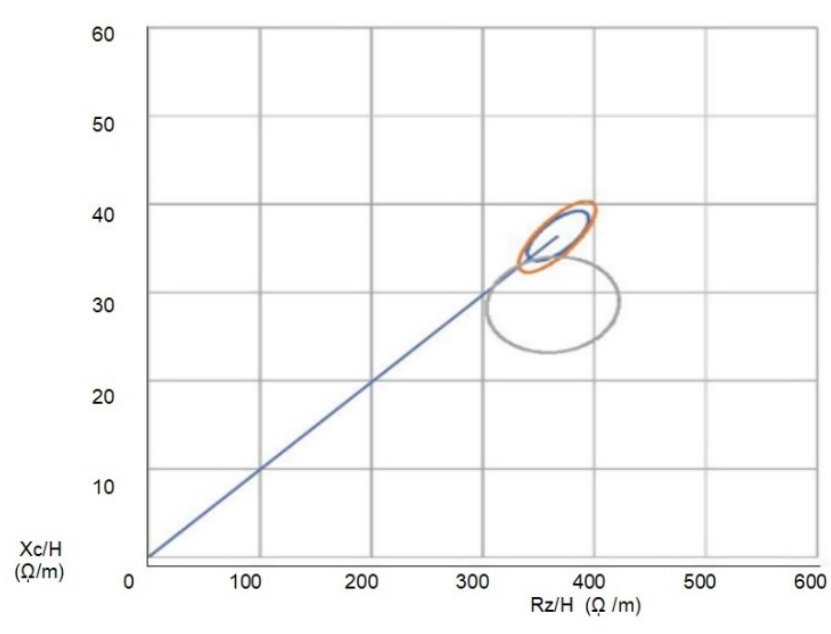

Fig.2: Female oncologic patients $\mathrm{R}+\mathrm{NR}$ at T0 and T6. Female oncologic patients at T0 (baseline) compared to T6 (after 6 months): R are marked by orange color and NR by grey color.

\section{Discussion}

This study focuses on the body composition assessment in oncologic patients who have been given a dietary treatment in order to prevent neoplastic cachexia. Our findings demonstrate that in oncologic male patients, both $\mathrm{R}$ and NR, at time zero and after 3 months, there is no substantial change in body composition. These data is also confirmed by the overlapping of each confidence interval.

However, comparing the two R and NR groups after 6 months, it is clear that the former maintains almost the same confidence interval, unlike the latter whose interval increases, indicating a light worsening both in the body composition and in the clinical conditions.

Besides, the same male responder group shows an improvement in the $\mathrm{PhA}$, (a positive prognostic factor) with an average weight loss between $2.6-3 \mathrm{~kg}$ of fat mass (FM).

Also, the female oncologic patients, $R$ and NR, do not show significant body composition changes at TO and T3. Data also show that the $\mathrm{R}$ female group maintains the confidence interval unlike the female NR group, whose confidence interval remarkably increases, therefore indicating an important alteration of body composition and subsequently a clinical conditions worsening.

In addition, comparing the NR male group with the NR female group after 6 months, a remarkable worsening of body composition (marker of tissue decay and damage) can be noticed in the latter.

Thus, both groups $\mathrm{R}$ and NR do not show progression toward sarcopenia after 3 months, while after 6 months there was muscle loss and a worsening in body composition in the NR group; this decay proved to be more expressed in female NRs, probably because of their sex-related genetically determined minor muscular strength and inferior muscle percentage.

In another study, a retrospective analysis also showed a lower rate of sarcopenia in responders to immunotherapy 
compared to non-responders - the disease control rates were obviously lower in patients with sarcopenia than in those without sarcopenia. Patients with sarcopenia exhibited a significantly shorter median progression-free survival than non-sarcopenia patients. Patients with sarcopenia were associated with poor outcomes for immunotherapy among those with advanced non-small cell lung cancer [17].

Weight loss and malnutrition remain a central concern for patients at all stages of treatment for cancer. The inability to maintain body weight is recognized as a poor prognostic factor in sustaining therapeutic response to neoadjuvant or adjuvant treatment and long-term survival.

There has recently been increased interest in the assessment of body composition in cancer patients for the purpose of nutritional evaluation and prognostication. However only few studies assessed its potential implication upon early and late outcomes. A systematic literature search was recently conducted for studies describing the assessment of body composition in patients with esophageal and gastric cancer: 28 studies (10 studies of them used BIA, 18 studies used computerized tomography) reported the assessment of body composition in 3193 patients: Sarcopenic patients had a higher incidence of postoperative major complications [18].

Meta-analysis of six studies presenting long-term outcomes identified worse survival in patients who were sarcopenic $[18,19]$ and higher rates of morbidity and inhospital mortality in sarcopenic patients prior to surgery; and an association between sarcopenia and reduced overall, recurrence-free and cancer-specific survival in patients [19].

A very recent study [20] evaluated the influence of sarcopenia in patients with locally advanced, not metastasized cancer undergoing curative treatment (perioperative chemotherapy and surgery) on morbidity and mortality in order to identify patients in need for nutritional intervention: sarcopenic patients were significantly older than nonsarcopenic patients (mean age 65.1 years), terminated the chemotherapy significantly earlier and showed higher Clavien-Dindo scores, indicating more severe perioperative complications, and they had a significantly shorter survival than non-sarcopenic patients [20].

In conclusion, the study points out to the positive influence of body composition assessment with a correct nutritional program for neoplastic cachexia prevention and treatment, despite the limited period of time and number of examined subjects.

To date, this turns out to be the most effective kind of approach in order to maintain body weight and, consequently, diminish the risk of developing the relative symptoms - this risk would be accompanied by a global worsening of oncologic patient conditions and by a possible loss of efficacy of cancer treatments.

The assessment of body composition has the potential to become a clinically useful tool that could support decisionmaking in patients suffering from cancer.
Therefore, a multidimensional assessment of oncologic patient is universally recognized as necessary in order to avoid malnutrition consequent to the disease. For this purpose, it is very clear that specialized hospital facilities and still more, multidisciplinary medical team targeting a better quality of patient's life, are increasingly needed.

\section{Conflict of interest}

Authors state no conflict of interest.

\section{References}

1. Planas M, Alvarez-Hernandez J, Leon-Sanz M, Celaya-Perez S., Araujo K, Garcia De Lorenzo A. Prevalence of hospital malnutrition in cancer patients: a sub-analysis of the PREDyCES $^{\circledR}$ study. Support Care Cancer. 2016; 24:429-435. https://doi.org/10.1007/s00520-015-2813-7

2. Arends J., Baracos V., Bertz H, F. Bozzetti, Calder P.C., Deutz N.E.P, Erickson N., Laviano A., Lisanti M.P., Lobo D.N., Mcmillan D.C., Muscaritoli M., Ockenga J., Pirlich M., Strasser F., De Van Der Schueren M. ESPEN expert group recommendations for action against cancer-related malnutrition, Clinical Nutrition, 2017; 36 (5): 1187-1196. https://doi.org/10.1016/j.clnu.2017.06.017

3. Fearon K.C., Voss A.C., Hustead D.S. Definition of cancer cachexia: effect of weight loss, reduced food intake, and systemic inflammation on functional status and prognosis. Am J Clin Nutr 2006; 83: 1345-1350. https://doi.org/10.1093/ajen/83.6.1345

4. Cederholm T., Barazzoni R., Austin P., Ballmer P., Biolo G., Bischoff S.C., Compher C., Correia I., Higashiguchi T., Holst M., Jensen G.L., Malone A., Muscaritoli M. ESPEN guidelines on definitions and terminology of clinical nutrition. Clin Nutr. 2017; 36:49-64. https://doi.org/10.1016/j.clnu.2016.09.004

5. Lochs H., Allison S.P., Meier R., Pirlich M., Kondrup J., Schneider S., Van Den Berghe G., Pichard C. Introductory to the ESPEN guidelines on enteral nutrition: terminology, definitions and general topics. Clin Nutr. 2006;25(2): 180-186. https://doi.org/10.1016/j.clnu.2006.02.007

6. Blauwhoff-Buskermolen S., Ruijgrok C., Ostelo R., De Vet H., Verheul H., De Van Der Schueren M. The assessment of anorexia in patients with cancer: Cut-off values for the FAACTA/CS and the VAS for appetite. Support Care Cancer 2016;24:661-666. https://doi.org/10.1007/s00520-015-2826-2

7. Hebuterne X., Lemarie E., Michallet M., De Montreuil C.B., Schneider S.M., Goldwasser F. Prevalence of malnutrition and current use of nutrition support in patients with cancer. JPEN J Parenter Enteral Nutr. 2014;38:196-204. https://doi.org/10.1177/0148607113502674

8. Pressoir M., Desne S., Berchery D., Rossignol G., Poiree B., Meslier M., Traversier S., Vittot M., Simon M., Gekiere J.P., Meuric J., Serot F., Falewee M.N. Prevalence, risk factors and clinical implications of malnutrition in French Comprehensive Cancer Centres. Br J Cancer. 2010;102:966-971.

https://doi.org/10.1038/sj.bjc.6605578 
9. Hamaker ME, van Huis-Tanja LH, Rostoft S. Optimizing the geriatrician's contribution to cancer care for older patients. J Geriatr Oncol. 2019 (in press). https://doi.org/10.1016/j.jgo.2019.06.018

10. Martin L., Senesse P., Gioulbasanis I. Diagnostic criteria for the classification of cancer-associated weight loss. J Clin Oncol 2015; 33: 90-9.

11. Del Fabbro E. Current and future care of patients with the cancer anorexia-cachexia syndrome. Am Soc Clin Oncol Educ Book 2015; e229-37.

https://doi.org/10.14694/edbook_am.2015.35.e229

12. Gonzalez M.C., Barbosa-Silva T.G., Bielemann R.M., Gallagher D., Heymsfield S.B.: Phase angle and its determinants in healthy subjects: influence of body composition Am J Clin Nutr. 2016;103(3):712-6. https://doi.org/10.3945/ajen.115.116772

13. Bosy-Westphal A., Danielzik S., Dörhöfer R.P., Later W., Wiese S., Müller M.J. Phase angle from bioelectrical impedance analysis: population reference values by age, sex, and body mass index. JPEN 2006;30:309-316. https://doi.org/10.1177/0148607106030004309

14. Lee S.Y., Lee Y.., Yang J.H., Kim C.M., Choi W.S. The Association between Phase Angle of Bioelectrical Impedance Analysis and Survival Time in Advanced Cancer Patients: Preliminary Study. Korean J Fam Med 2014;35:251-256. https://doi.org/10.4082/kjfm.2014.35.5.251
15. Gupta D., Lammersfeld C.A., Vashi P.G., King J., Dahlk S.L., Grutsch J.F. Bioelectrical impedance phase angle as a prognostic indicator in breast cancer. BMC Cancer 2008;8:249. https://doi.org/10.1186/1471-2407-8-249

16. Muscaritoli M. Perspectives of health care professionals on cancer cachexia: results from three global surveys. Ann Oncol. 2016;27(12):2230-2236. https://doi.org/10.1093/annonc/mdw420

17. Nishioka N., Uchino J., Hirai S.Association of Sarcopenia with and Efficacy of Anti-PD-1/PD-L1 Therapy in Non-Small-Cell Lung Cancer. J Clin Med. 2019;8(4):450. https://doi.org/10.3390/jcm8040450

18. Boshier P.R., Henegan R., Markar S.R., Baracos V.E., Low D.E. Assessment of body composition and sarcopenia in patients with esophageal cancer: a systematic review and metaanalysis. Diseases of the Esophagus. 2018;31(8):1040-47. https://doi.org/10.1093/dote/doy089.ps02.248

19. Correia MI, Waitzberg DL. The impact of malnutrition on morbidity, mortality, length of hospital stay and costs evaluated through a multivariate model analysis. Clin Nutr. 2003;22(3):235-9. https://doi.org/10.1016/s0261-5614(02)00215-7

20. Koch C., Reitz C., Schrekenbach T. Sarcopenia as a prognostic factor for survival in patients with locally advanced gastroesophageal adenocarcinoma. Plos One 2019;14(10):e0223613. https://doi.org/10.1371/journal.pone.0223613 